\title{
Minimizing nocebo effect: Pragmatic approach
}

Majed Chamsi-Pasha, Mohammed Ali Albar ${ }^{1}$, Hassan Chamsi-Pasha ${ }^{2}$

Departments of Medicine and ${ }^{2}$ Cardiology, King Fahd Armed Forces Hospital, ${ }^{1}$ Department of Medical Ethics, International Medical Center, Jeddah, Saudi Arabia

\begin{tabular}{|c|}
\hline Access this article online \\
\hline Website: www.avicennajmed.com \\
\hline DOI: 10.4103/ajm.AJM_59_17 \\
\hline Quick Response Code: \\
\hline
\end{tabular}

\begin{abstract}
The nocebo effect, the inverse of the placebo effect, is a well-established phenomenon, yet under-appreciated. It refers to nonpharmacological, harmful, or undesirable effects occurring after active or inactive therapy. The frequency of adverse events can dramatically increase by informing patients about the possible side effects of the treatment, and by negative expectations on the part of the patient. Patients who were told that they might experience sexual side effects after treatment with $\beta$-blocker drugs reported these symptoms between three and four times more often than patients in a control group who were not informed about these symptoms. Nocebo effect has been reported in several neurological diseases such as migraine, epilepsy, multiple sclerosis, Parkinson's disease and neuropathic pain, and in patients with depression. The investigation of the biological and theoretical underpinning of the nocebo phenomenon is at an early stage, and more research is required. Physicians need to be aware of the influence of nocebo phenomenon and be able to recognize it and minimize its effects.
\end{abstract}

Key words: Drugs, medical ethics, nocebo, placebo, treatment

\section{INTRODUCTION}

A placebo or nocebo effect occurs when an inert substance creates a beneficial or harmful effect in a person who takes it. Similarly, a nocebo response can occur with an inert or noninert substance as a worsening of the diagnosed condition or as treatment-emergent adverse effects. ${ }^{[1]}$ While the placebo effect has been well known for a long time, much less is known about its negative counterpart, named the "nocebo" effect, despite the fact that it may be a significant factor affecting the clinical outcomes.

The placebo effect (from the Latin, "I shall please") describes a desired clinical effect that arises solely from the patient's positive expectations. A typical example is giving a physiologically inert agent, to a cohort of subjects who reveal the same benefit as a similar cohort who is given an active drug. On the contrary, the nocebo effect (from Latin "I will harm") refers to the induction or worsening of symptoms induced by sham or active therapies. ${ }^{[2]}$ The term nocebo was coined by Walter Kennedy in 1961 to denote the

Address for correspondence: Dr. Majed Chamsi-Pasha, Department of Medicine, King Fahd Armed Forces Hospital, P. O. Box: 9862, Jeddah 21159, Saudi Arabia.

E-mail: majed.chamsi.pasha@hotmail.com counterpart to the use of placebo. The nocebo effect reflects changes in human psychobiology involving the brain, body, and behavior rather than drug toxicity. ${ }^{[3]}$

Nocebo effects, which have been encountered in both clinical and research settings, occur when negative effects arise out of a patient's or participant's negative expectations. ${ }^{[4]} \mathrm{A}$ study involving the use of finasteride in patients with benign prostatic hypertrophy revealed a 3 -fold increase in the incidence of erectile dysfunction (ED) when this potential side effect was disclosed to the patient. ${ }^{[5]}$ In a similar study involving the use of beta-blocker (atenolol), only $3.1 \%$ of men reported (ED) when they were not told the name of the medication, $15.6 \%$ when they were told the drug name but not the potential risk of ED, while the incidence was $31.2 \%$ when they were told both the drug name and the potential risk of ED. ${ }^{[6]}$ Furthermore, Schweiger and Parducci reported that more than two-thirds of a sample including

This is an open access article distributed under the terms of the Creative Commons Attribution-NonCommercial-ShareAlike 3.0 License, which allows others to remix, tweak, and build upon the work non-commercially, as long as the author is credited and the new creations are licensed under the identical terms.

For reprints contact: reprints@medknow.com

Cite this article as: Chamsi-Pasha M, Albar MA, Chamsi-Pasha H. Minimizing nocebo effect: Pragmatic approach. Avicenna J Med 2017;7:139-43. 
34 college students reported mild headaches when they were told that a (nonexistent) electric current was passing through their skulls. ${ }^{[7]}$

These studies and many others, indicate that, in certain kinds of side effects, disclosing a potential adverse event (AE) significantly increases the likelihood of such an event occurring. Obtaining patient consent is fundamentally important in clinical practice. However, when adverse effects occur directly as a result of disclosure, clinicians are faced with an ethical dilemma of two competing principles: autonomy and nonmaleficence and hence the importance of how to minimize the nocebo effects without encroaching on the principle of autonomy.

\section{WHAT IT MEANS IN CLINICAL PRACTICE?}

\section{Neurological diseases}

Nocebo effect is very prevalent among neurological diseases resulting in low adherence and treatment outcome. The most productive models to better understand the neurobiology of the placebo effect are pain and Parkinson's disease. ${ }^{[8]}$ In these medical conditions, several neurotransmitters have been identified. Studies in the field of pain propose three mechanisms to explain the nocebo effect: expectation, conditioning, and anticipatory anxiety. Expectation is evident when the person expects that the drug will fail or not work. The conditioning mechanism is evident when the patient experienced negative results with previous other drugs. ${ }^{[9]}$

\section{Nocebo effect in headache}

Most of the studies involving nocebo effect were performed in randomized, controlled, clinical trials treatments that document harmful side effects and dropouts in the control group. The frequency of nocebo was estimated by the percentage of placebo-treated patients reporting at least one adverse side effect. The dropout frequency was estimated by the percentage of placebo-treated patients who discontinued the treatment due to intolerance. In studies of symptomatic treatment for migraine, the nocebo and dropout frequencies were $18.45 \%$ and $0.33 \%$, but rose to $42.78 \%$ and $4.75 \%$ in preventative treatment studies. In trials for prevention of tension-type headache, nocebo and dropout frequencies were $23.99 \%$ and $5.44 \%$. For symptomatic treatment of a cluster headache, the nocebo frequency was $18.67 \%$ Table $1 .{ }^{[10]}$

\section{Nocebo effect in neuropathic pain}

Neuropathic pain affects about $8 \%$ of the world population and is considered by many authorities an endemic disease. In a meta-analysis published by Papadopoulos and Mitsikostas on the nocebo effects in neuropathic pains trials, the frequency of nocebo effect was $52 \%$, and the dropout rate by nocebo effect was $6 \% .{ }^{[1]]}$

\section{Nocebo effect in epilepsy}

Several meta-analytic approaches have investigated the extent of placebo and nocebo response in randomized, placebo-controlled, clinical trials of epilepsy. Significant differences have been identified in the AEs-induced nocebo effect across different conditions. ${ }^{[12]} \mathrm{A}$ recent meta-analysis of placebo-controlled clinical trials in patients on antiepileptic therapy showed that three out of five placebo-treated patients (60.8\%) reported at least one $\mathrm{AE}$ and $4.0 \%$ discontinued placebo treatment as a result. ${ }^{[13]}$

\section{Other neurological diseases}

In a meta-analysis of 3544 placebo-treated patients with Parkinson's disease, $64.7 \%$ reported at least one AE and $8.8 \%$ discontinued placebo treatment due to intolerance. ${ }^{[14]}$ A meta-analysis of the rate of nocebo responses in patients with restless legs syndrome showed that the pooled nocebo response was $45.36 \% .{ }^{[15]}$ Nocebo responses in multiple sclerosis trials are substantial and appear to have increased significantly in recent years. The pooled incidence of nocebo responses was $74.4 \%$ Table $1 .{ }^{[16]}$

\section{Statin-associated muscle symptoms}

Statin intolerance, predominantly due to statin-associated muscle symptoms (SAMS), is a common and difficult-to-manage condition affecting millions of patients worldwide. A few reports suggest that SAMS might result from patients' perceptions about statins in light of negative press reports of statin use, or even poor understanding of warnings about statin-associated adverse effects. ${ }^{[3]}$ A recent analysis of The Anglo-Scandinavian Cardiac Outcomes Trial population $^{[17]}$ showed that fewer patients could possibly report SAMS with statins if they receive the drug blindly than if they receive it as an open label, or they might have SAMS even if they received a placebo, indicating a highly improbable pharmacological basis and possible contribution of nocebo effect. ${ }^{[3,17]}$

\section{Nocebo effect in depression}

Among antidepressant trials, the size of both placebo and nocebo reactions varied substantially. ${ }^{[18]}$ The average rate of AEs and related discontinuations in placebo groups were $57 \%$ and $4 \%$, respectively. When informing patients about possible AEs, physicians should avoid inducing negative expectations and hence, nocebo reactions, particularly when working with male patients and those with early onset depression. ${ }^{[19]}$ 


\section{Chronic pain after surgery}

Chronic pain after major surgery is a common problem, estimated to occur in $20 \%-50 \%$ of patients. ${ }^{[20]}$ The principle of informed consent compels the clinician to explain possible side effects, such as chronic postsurgical pain, before performing surgeries. The honest disclosure may itself induce adverse effects through expectancy mechanisms (nocebo effects), such as persistent postsurgical pain, contradicting the principle of nonmaleficence. Ruan and Kaye propose that for surgeries which are lifesaving, or for major functional restoration, such as tumor removal, trauma surgical intervention, and so on, where postsurgical pain is of less concern comparatively, disclosure of the likelihood of chronic postsurgical pain in a balanced manner may be warranted. ${ }^{[20]}$

\section{CONTRIBUTING FACTORS}

The biological basis of the nocebo effect is only now beginning to be disentangled. The drivers of the placebo and nocebo phenomena may be a synergy of multiple biological and psychological variables, mediated by a further multitude of contextual and individual variables. There is clear evidence of physiological factors that underpin the phenomena, as well as a contribution by psychological factors. ${ }^{[21]}$

Patient expectations of treatment side effects can have a significant influence over the type and number of symptoms that are reported following medical therapy. Expectations may be formed as a result of the informed consent process, through observation of another person experiencing symptoms, and through information presented in the media. Commonly held negative perceptions about generic drugs may result in the expectation of more adverse effects, and thus more treatment side effects. ${ }^{[2,23]}$ The public and health-care providers often hold negative views of the generic medicines. Many patients do not trust generic drugs, and consider them as being of lower quality and less effective than their brand name counterparts. ${ }^{[24]}$ Many physicians and pharmacists also view generic drugs as being less safe, of lower quality, and more likely to cause side effects even though these views are not supported by randomized controlled studies. ${ }^{[23,25]}$

Besides, conditioning and expectancy affect nocebo responses, although the size of their effect vary greatly, depending on interindividual differences and different experimental paradigms. The neurobiology of the nocebo phenomenon emphasizes the involvement of reward pathways, such as the $\mu$-opioid and dopamine pathways. Neurobiological pathways have been investigated in a limited range of experimental paradigms. The interconnection of psychological and physiological drivers of nocebo responses is a core feature of this phenomenon and further research is needed to fully understand the underpinnings of this phenomenon. ${ }^{[21]}$ Recognition of the nocebo-driven adverse effects presents a challenge to the physicians in their daily practice, because of its nonspecific nature and the similarity to the active medication's expected profile. Traits such as type A personality, neuroticism, and pessimism may predispose individuals to nocebo effect. ${ }^{[26]}$ News media play an important role in the development and spread of symptoms during health scares. The presentation of patient case studies in the news media is likely to be problematic, as both contribute to increased expectations of negative treatment outcomes. ${ }^{[23]}$

\section{CLINICAL MANAGEMENT}

Clinical management of the nocebo effect includes recognition of the phenomenon, changing the way of disclosure of potential drug-related adverse effects, shaping the patients' expectations, and reducing exposure to people experiencing these side effects.

Physicians could reduce expectations of symptoms, especially for persons with at-risk personality types, though determining exactly which personality types these are, requires further research. These suggestions may have some limitations in terms of consent and paternalism, but there is always a scope to develop innovative ways to reduce nocebo effects without withholding information. ${ }^{[22]}$

With the litigious environment back in mind, doctors may over-emphasize the risks of treatment for medicolegal reasons, which can increase the risk of inducing the nocebo effect. In the clinical setting, this may compromise therapy, and in the context of informed consent for clinical trials, may increase the rate of $\mathrm{AE}$ reporting. ${ }^{[2]]}$

Wells and Kaptchuk advocate that the conflict between the informed consent and nonmaleficence might be resolved by recognizing that adverse effects have no clear black or white "truth." They have proposed a practical approach to minimize nocebo effects, while still maintaining patient's autonomy through "contextualized informed consent," where disclosed information is tailored in a way that will reduce expectancy-induced adverse effects while preserving the respect of patient's autonomy and truth-telling. ${ }^{[27]}$ The notion of contextualized consent should consider how much information a patient wants to know with a proper compromise between patient's autonomy and unrealistic paternalism. 
Del Pozo and Fins ${ }^{[28]}$ emphasized that the Western way of obtaining informed consent in a patient from the Eastern culture may provide "too much information and may leave the patient feeling misinformed." Giving too much information, at times, may raise suspicion that the physician might be withholding information or even hiding the truth. ${ }^{[28,29]}$ To respect the autonomy of the patient, the physician should be fully aware of the cultural values and behaviors of his/her patient.

Although it is a great challenge to discuss the risks of therapy without creating anxiety, it is possible to engage patients in conversations that respect autonomy, avoid undue paternalism, and recognize that serious side-effects will be disclosed regardless. ${ }^{[23]}$

While delivering the essential information, it would be preferable to bring attention to the benefits rather than losses. This method is called "framing" and in message framing, focus is placed on the percentage of patients who usually tolerate the treatment well or do not endure a particular adverse effect.

Shaping expectations may play a role in minimizing side effects. It is common, for example, for physicians to warn patients of the painful nature of an impending procedure, such as the administration of local anesthesia. However, this may have counterproductive effects, as it has been shown that lower scores for pain occur if the message was focused on the injection's beneficial effects, rather than it being painful. ${ }^{[26]}$

Other suggestions include placing more emphasis on patients' ability to cope with mild symptoms; permitted noninformation, in which a patient may agree to receive no information or less information about mild or temporary adverse effects; and patient education about nocebo effects including examples. ${ }^{[30]}$ Physicians should be aware of their own perceptions of generic drugs, and taking care not to transmit negative expectations about them to patients.

Finally, placebo and nocebo effects will always be present during the therapeutic process and may positively or negatively impact treatment effects (such as pain, incapacity, and satisfaction). Hence, it is up to the health professionals to make daily efforts searching for better therapeutic experiences for their patients. ${ }^{[31]}$ Clinicians, in their daily practice, should be aware that their presence in the therapeutic relationship has agency over and above the effects of the drugs they use or the procedures they perform. This is of paramount importance since it is a reminder that the context in which medical care is delivered
Table I: Nocebo frequency and dropouts in clinical trials

\begin{tabular}{lcc}
\hline Disease & $\begin{array}{c}\text { Nocebo } \\
\text { frequency (\%) }\end{array}$ & $\begin{array}{c}\text { Dropout } \\
\text { frequency (\%) }\end{array}$ \\
\hline Symptomatic treatment for migraine & 18.45 & 0.33 \\
Preventative treatment for migraine & 42.78 & 4.75 \\
Tension-type headache prevention & 23.99 & 5.44 \\
Epilepsy & 60.8 & 4.0 \\
Multiple sclerosis & 74.4 & $\mathrm{NA}$ \\
Parkinson's disease & 64.7 & 8.8 \\
Neuropathic pain & 52 & 6 \\
Restless legs syndrome & 45.36 & $\mathrm{NA}$ \\
Persistent depressive disorder & 57 & 4 \\
\hline NA: Not available & &
\end{tabular}

influences important patient outcomes including treatment compliance, the experience of adverse effects, and the efficacy of our care. ${ }^{[32]}$

\section{RECOMMENDATIONS}

Physicians should be able to recognize this phenomenon and master tactics on how to manage these effects to enhance the quality of clinical practice. Managing patients' beliefs and experiences are at the core of possible strategies. Creating a good physician-patient relationship, increasing empathic attitudes, exposing information suitably, decreasing expectations of adverse effects, and promoting social contact between successfully treated patients, may all contribute to minimizing nocebo effects. When the medical problem allows for a small delay in the start of therapy, a lower initial dose might be helpful. ${ }^{[33]}$

\section{CONCLUSION}

The nocebo effect is a common and clinically significant phenomenon. Nocebo responses may result from unintended negative suggestions about AEs of medications by physicians or nurses. Increased awareness of its existence, features, and developing strategies to manage are fundamental for physicians to mitigate its impact on clinical practice.

\section{Financial support and sponsorship}

Nil.

\section{Conflicts of interest}

There are no conflicts of interest.

\section{REFERENCES}

1. Dodd S. Current insights in the placebo and nocebo phenomena. Clin Ther 2017;39:456-7.

2. Planès $\mathrm{S}$, Villier $\mathrm{C}$, Mallaret $\mathrm{M}$. The nocebo effect of drugs. Pharmacol Res Perspect 2016;4:e00208.

3. Pedro-Botet J, Rubiés-Prat J. Statin-associated muscle symptoms: Beware of the nocebo effect. Lancet 2017;389:2445-6. 
4. Cohen S. The nocebo effect of informed consent. Bioethics 2014;28:147-54.

5. Mondaini N, Gontero P, Giubilei G, Lombardi G, Cai T, Gavazzi A, et al. Finasteride $5 \mathrm{mg}$ and sexual side effects: How many of these are related to a nocebo phenomenon? J Sex Med 2007;4:1708-12

6. Silvestri A, Galetta P, Cerquetani E, Marazzi G, Patrizi R, Fini M, et al. Report of erectile dysfunction after therapy with beta-blockers is related to patient knowledge of side effects and is reversed by placebo. Eur Heart J 2003;24:1928-32.

7. Schweiger A, Parducci A. Nocebo: The psychologic induction of pain. Pavlov J Biol Sci 1981;16:140-3.

8. Carlino E, Piedimonte A, Benedetti F. Nature of the placebo and nocebo effect in relation to functional neurologic disorders. Handb Clin Neurol 2016;139:597-606.

9. Bittar C, Nascimento OJ. Placebo and nocebo effects in the neurological practice. Arq Neuropsiquiatr 2015;73:58-63.

10. Mitsikostas DD, Mantonakis LI, Chalarakis NG. Nocebo is the enemy, not placebo. A meta-analysis of reported side effects after placebo treatment in headaches. Cephalalgia 2011;31:550-61.

11. Papadopoulos D, Mitsikostas DD. A meta-analytic approach to estimating nocebo effects in neuropathic pain trials. J Neurol 2012;259:436-47.

12. Zaccara G, Giovannelli F, Giorgi FS, Franco V, Gasparini S. Analysis of nocebo effects of antiepileptic drugs across different conditions. J Neurol 2016;263:1274-9.

13. Zis $\mathrm{P}$, Shafiq F, Mitsikostas DD. Nocebo effect in refractory partial epilepsy during pre-surgical monitoring: Systematic review and meta-analysis of placebo-controlled clinical trials. Seizure 2017;45:95-99.

14. Stathis P, Smpiliris M, Konitsiotis S, Mitsikostas DD. Nocebo as a potential confounding factor in clinical trials for Parkinson's disease treatment: A meta-analysis. Eur J Neurol 2013;20:527-33.

15. Silva MA, Duarte GS, Camara R, Rodrigues FB, Fernandes RM, Abreu D, et al. Placebo and nocebo responses in restless legs syndrome: A systematic review and meta-analysis. Neurology 2017;88:2216-24.

16. Papadopoulos D, Mitsikostas DD. Nocebo effects in multiple sclerosis trials: A meta-analysis. Mult Scler 2010;16:816-28.

17. Gupta A, Thompson D, Whitehouse A, Collier T, Dahlof B, Poulter N, et al. Adverse events associated with unblinded, but not with blinded, statin therapy in the anglo-scandinavian cardiac outcomes trial-lipid-lowering arm (ASCOT-LLA): A randomised double-blind placebo-controlled trial and its non-randomised non-blind extension phase. Lancet 2017;389:2473-81.

18. Dodd S, Schacht A, Kelin K, Dueñas H, Reed VA, Williams LJ, et al.
Nocebo effects in the treatment of major depression: Results from an individual study participant-level meta-analysis of the placebo arm of duloxetine clinical trials. J Clin Psychiatry 2015;76:702-11.

19. Meister R, Jansen A, Härter M, Nestoriuc Y, Kriston L. Placebo and nocebo reactions in randomized trials of pharmacological treatments for persistent depressive disorder. A meta-regression analysis. J Affect Disord 2017;215:288-98.

20. Ruan X, Kaye AD. Disclosure without or with reservation? contemplating the development of persistent postsurgical pain due to nocebo effect. Am J Surg 2016;212:1029-30.

21. Dodd S, Dean OM, Vian J, Berk M. A review of the theoretical and biological understanding of the nocebo and placebo phenomena. Clin Ther 2017;39:469-76.

22. Webster RK, Weinman J, Rubin GJ. A systematic review of factors that contribute to nocebo effects. Health Psychol 2016;35:1334-55.

23. Faasse K, Petrie KJ. The nocebo effect: Patient expectations and medication side effects. Postgrad Med J 2013;89:540-6.

24. Himmel W, Simmenroth-Nayda A, Niebling W, Ledig T, Jansen RD, Kochen MM, et al. What do primary care patients think about generic drugs? Int J Clin Pharmacol Ther 2005;43:472-9.

25. Hassali MA, Kong DC, Stewart K. A comparison between senior medical students' and pharmacy pre-registrants' knowledge and perceptions of generic medicines. Med Educ 2007;41:703-10.

26. Bishop FL, Coghlan B, Geraghty AW, Everitt H, Little P, Holmes MM, et al. What techniques might be used to harness placebo effects in non-malignant pain? A literature review and survey to develop a taxonomy. BMJ Open 2017;7:e015516.

27. Wells RE, Kaptchuk TJ. To tell the truth, the whole truth, may do patients harm: The problem of the nocebo effect for informed consent. Am J Bioeth 2012;12:22-9.

28. Del Pozo PR, Fins JJ. Islam and informed consent: Notes from Doha. Camb Q Healthc Ethics 2008;17:273-9.

29. Chamsi-Pasha H, Albar MA. Western and Islamic bioethics: How close is the gap? Avicenna J Med 2013;3:8-14.

30. Häuser W, Hansen E, Enck P. Nocebo phenomena in medicine: Their relevance in everyday clinical practice. Dtsch Arztebl Int 2012;109:459-65.

31. Fagundes FR, Reis FJ, Cabral CM. Nocebo and pain: Adverse effects of excessive information. Rev Dor São Paulo 2016;17:157-8.

32. Arnold MH, Finniss DG, Kerridge I. Medicine's inconvenient truth: The placebo and nocebo effect. Intern Med J 2014;44:398-405.

33. Chavarria V, Vian J, Pereira C, Data-Franco J, Fernandes BS, Berk M, et al. The placebo and nocebo phenomena: Their clinical management and impact on treatment outcomes. Clin Ther 2017;39:477-86. 